\title{
Mathematical modeling and methods of recognition of plant objects
}

\author{
Irina Mayatskaya ${ }^{1^{*}}$ \\ ${ }^{1}$ Don State Technical University, 1, Gagarin sq., 344003, Rostov-on-Don, Russia
}

\begin{abstract}
The purpose of the study - to show the basic model plant objects that are based on different branches of mathematics methods: analytical geometry, surface theory, graph theory and fractal geometry, as well as methods for recognizing these objects these objects. The development of mathematical methods and computer technologies brings mathematical modeling of complex objects and processes in which they participate to a new level.
\end{abstract}

\section{Introduction}

Currently, there is interest in the mathematical modeling of natural objects and, in particular, to the modeling of plant objects. By studying the models of plant materials, it is possible to build a model of the technological process of collecting and processing of grain and other agricultural crops using robotic complexes. And immediately the problem of recognizing agricultural plants arises [1-5].

The development of mathematics and computer technologies brings mathematical modeling of complex processes involving plant objects to a new level. The task is to use more extensively mathematical methods for creating models of plant objects and more accurate recognition of plants with a fuzzy outline.

\section{Features of mathematical modeling of plant objects}

Mathematical modeling of plant objects opens up prospects for the creation of robotic complexes for harvesting and processing grain and other agricultural crops. Knowledge of the models of these objects is also necessary for the computer study of technological processes in which plant materials are involved $[1,2,6]$. To build these models, you need to take into account the complexity of their structure, the real shape of the object and the ability to make adjustments.

When creating models of plant objects, necessary satisfy the following conditions:

1. the degree of knowledge of the form of a plant object and mathematical methods for its description;

2. the acceptability of the restrictions and assumptions that arose in the formulation of the problem under study;

\footnotetext{
* Corresponding author: irina.mayatskaya@mail.ru
} 
3. taking into account the complexity of the plant object and its specific properties;

4. the convenience of using this mathematical method;

5. the possibility of introducing empirically obtained information into the mathematical model of a plant object, the possibility of introducing corrections for parameters;

6. application of the obtained models in mathematical modeling of technological processes;

7. application of mathematical methods for recognizing these objects.

There are four main directions in the construction of mathematical models of plant objects $[1,2]$ :

1. analysis of a discrete or continuous medium, which is formed by a large number of plants or their parts, for example, a grain heap;

2. construction of models of individual plants;

3. construction of models of individual vegetative parts of plants, for example, grains, seeds, stems, leaves, fruits, roots;

4. models of plant microstructures, for example, plant cells.

In this study, attention is paid to the third direction, the application of mathematical methods in modeling and recognition of individual parts of a plant object [1-6].

Studying the laws of the structure of plant facilities, we can improve not only the processes of collection and processing, but also a wide variety of structures, including composite, by using the principles of the structure of biological systems. This trend makes it possible to introduce the practice of structural design features of the structure of plants, developing a different direction - bionics.

\section{Mathematical methods for modeling plant objects}

Mathematical modeling is an effective method for researching a given problem. Using the methods of this research with using software systems and creating a computer model for a complex system, it is possible to select the optimal parameters of this technological process.

When modeling plant objects, a wide variety of mathematical methods are used, for example, analytical geometry, fractal geometry, surface theory and graph theory. Depending on the task, you can choose one or another solution. Methods of analytical geometry are suitable for constructing models of seeds of agricultural crops and leafy materials $[3,4]$.

The following models can be used to build models of seeds of various crops:

1.elliptical with a cross-section in the form of a Pascal snail or cardioid (wheat, rye);

2. ellipsoid (wheat, rye, beans);

3. spindle-shaped asymmetric with a cross-section in the form of a Pascal snail or cardioid (barley);

4. spindle-shaped asymmetric with an elliptical cross-section (oats);

5. spindle-shaped symmetric with a cross-section in the form of a Pascal cochlea or a cardioid or an ellipse;

6.lenticular (lentils, peas);

7. a curvilinear pyramid with a cross-section in the form of a rhombus, a curved triangle, a rhombus, a curved quadrangle (sunflower, buckwheat, corn);

8.beans (beans).

For each model, analytical structures are obtained that describe the surface of these models and the main mechanical and mathematical characteristics. In order to construct models of grains, seeds, fruits, and the leaves applied methods of analytical geometry. On the surface of the object supporting section allocated with subsequent analytical description of the functions, which are laid as a basis in the model under construction surface or 
contour. When determining the shape of the sheet, you can use the envelope contour and equations describing curved polygons.

When constructing models of leafy materials, structures with three types of branching of shoots (Fig. 1) were used [5]:

1. dichotomous;

2. monopodial (symmetrical and asymmetrical);

3.sympoidal.

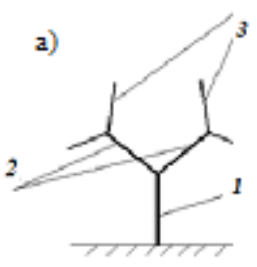

b)

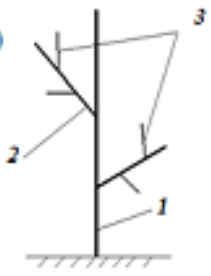

c)

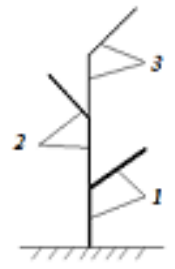

Fig. 1. Types of branching of shoots (1 - axis of branching of the 1st order of branching, 2 - axis of the 2 nd order of branching, and 3 - axis of the 3rd order of branching): a - dichotomous, $b$ monopodial, c - sympodial.

And also models can be with a branchy structure from the beginning of the stem, from the stem itself with a "straightened" structure, or have the appearance of a creeping or climbing plant (Fig. 2, 3). The branches can be either straight or curved, for example, in the form of parabolas with a top and without a top. The choice of the method for describing the model depends on the specific technological process. The most commonly used methods are analytical geometry.

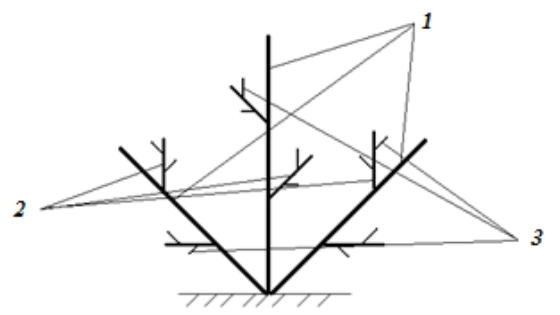

Fig. 2. Model with a branched structure from the beginning of the stem.

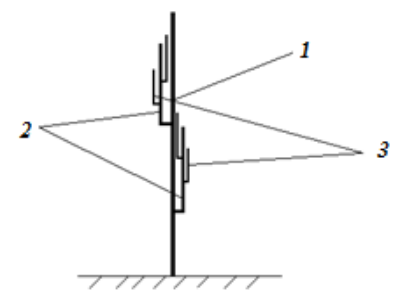

Fig. 3. Model with a "straightened" structure.

When modeling leafy materials, the following principles of the internal structure of stems should be taken into account:

1) the principle of joint work of structural components that ensure high reliability of functioning; 
2) the principle of "reinforcement", which characterizes the quantity, location and shape of mechanical tissue;

3) the principle of structural construction. The stems of some plants are characterized by reinforcing fabric, which is a frame for strengthening the fibers.

Let's consider other methods of mathematics: fractal geometry and graph theory [8-11]. The choice of mathematical methods depends on the specific technological process, the set research goal and the capabilities of the software.

To build a graph model of a plant object, networks are used (Fig. 4). When constructing the skeletal structure of leafy plants of all types of branching, matrices of edges and nodes are used. The graph can be drawn on a plane or in three-dimensional space. It can be displayed as a whole, in part, pulling some subgraphs into vertices that can be disclosed. Subgraphs are fruits, seeds, ear, leaves and other vegetative organs of the plant.

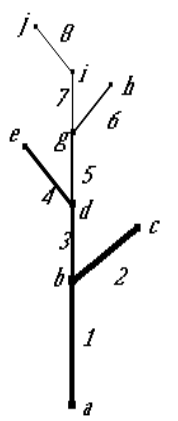

Fig. 4. Plant model with sympodial branching in the form of a graph.

Analyzing the structure of the matrix edges and nodes of graph-model type sympodial branching, it can be noted that they are almost quasi-diagonal matrices. In off-diagonal blocks, all elements are zero, and the diagonal blocks have the same structure. For the graph model shown in Fig. 5, these blocks are the same (Fig. 5, a). Then for a graph model with a dichotomous type branching in blocks, the elements have a similar structure, as if diverging from the zero diagonal (Fig. 5, b).
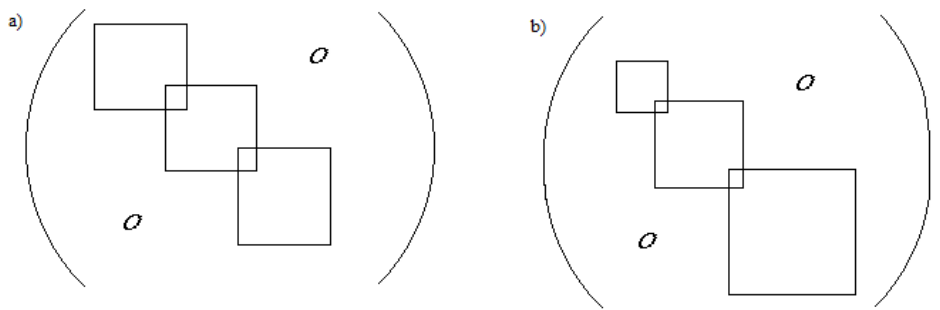

Fig. 5. Matrix structure for a plant with sympodial (a) and dichotomous (b) branching types.

With the first model, it is possible to describe cereal crops, and with the help of the second, branch crops. For cereals, the ear can also be described using graph theory and then another diagonal block is added (Fig. 6, a). For legumes, we introduce nonzero elements into the matrix to determine the position of the bean, which can be located throughout the plant (Fig. 6, b). These models of leafy materials create a theoretical model of the technological process considered as graphs [9]. 

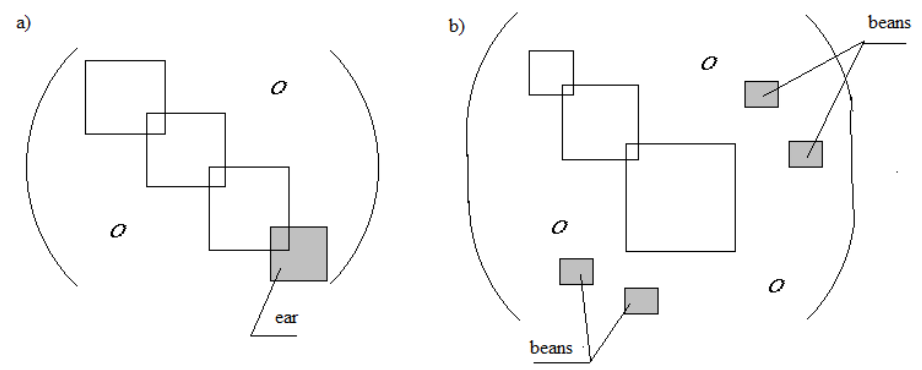

Fig. 6. Matrix structure for cereals and legumes with sympodial (a) and dichotomous (b) branching types.

For modeling of the leafy plants by fractal geometry methods convenient to use iterative system functions. This method is based on sequential iterative calculation of the coordinates of new points using the coordinate transformation function in space, which determines the shape of the fractal.

This method of construction of models of objects involves the use of vegetable iterated function systems which can be described as a sequential iterative calculation of the new coordinate points in space: $x_{k+1}=F_{x}\left(x_{k}, y_{k}\right)$ and $y_{k+1}=F_{y}\left(x_{k}, y_{k}\right)$, where $F_{x}, F_{y^{-}}$ the function of coordinate transformation, which define the shape of a fractal. It is convenient to use when constructing various leafy plants. It is necessary to choose the generalized analytical model of the plant structure that would most accurately describe the structure of the plant. In fig. 7 shows models using iterative functions. Depending on the iteration number, you can set the segment thickness, color, cross-sectional shape, and the shape of the leaves. In fig. 7 presented fractals, which are plants with linearly reduced branches.

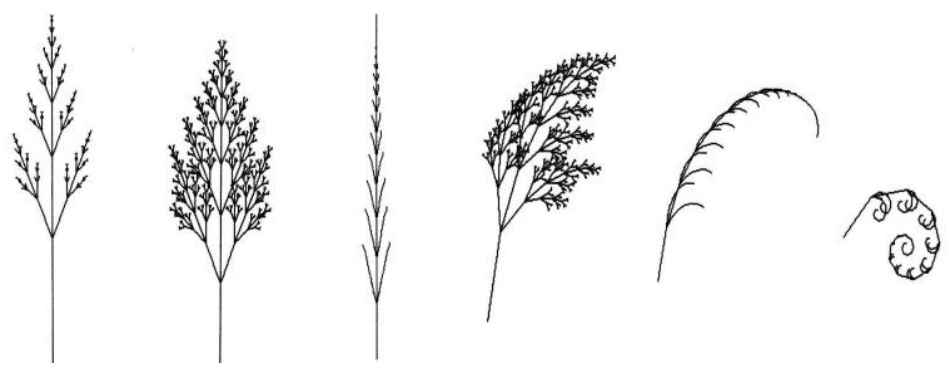

Fig. 7. Examples of building symmetrical models using iterative functions.

When modeling using fractals, it is necessary to find some rules by which the structure of the plant itself is built. On the number of iterations it depends on the structure of the model. The property of self-similarity found in nature very often, for example, the coast line, the crown of the tree. To describe the fractal model of a plant, it is necessary first of all to establish the relationship between the final result and the given initial structure of the plant as the dynamic law: $x_{k+1}=f\left(x_{k}\right)$. Finding this dependence can be very difficult. You can use not only the real numbers, but also complex. Only linear dependences of these functions with real numbers are considered. You can take a function in the form of a parabola, or another curve close to the real object. The disadvantage of this method is that the lengths of straight or curved line segments will be proportional and decrease with each iteration. 
Fractal geometry capabilities allow you to build plant models with complex geometry, creating the most amazing forms. The difficulty of this method consists in the selection of these functions, the disadvantage is also the necessity of determining the proportions in the geometric structure of the plant. Although using of fractals to describe some plants give a fairly accurate description of the object.

Fractal geometry methods are now widely used by researchers in the widest areas of science and technology. The advantages of this method when modeling plant objects are not immediately visible. It is necessary to further study the possibilities of using a wide variety of mathematical methods in modeling both in the processes of harvesting and processing plant materials, and in the study of theoretical problems.

\section{Recognition methods of plant objects}

The problem of recognizing images of plant objects includes three stages: description of plant objects and construction of their models; development, research and optimization of the choice of mathematical methods for information processing and analysis of the obtained images; software implementation of mathematical recognition methods.

In this case, you can solve the following tasks:

1.informational, that is, it is necessary to bring plant objects to some standard description and to determine to which class the object belongs;

2. approximate, that is, introduce the concept of similarity between plant objects and evaluate to which class the object under consideration is closer;

3. comparative, that is, it is necessary to have descriptions of plant objects and compare the object with already known classes;

4. non-formalized, that is, it is difficult to apply mathematical methods for certain plant objects;

5.with poor initial information, i.e. incomplete, probabilistic and other description of the plant object.

When studying the processes of recognition of plant objects themselves, it is necessary to carry out a detailed analysis of their structure [12-14]. If the real object does not have a clear outline or image, then you can recognize it by indirect signs, analyzing the entire plant. The study is devoted to the recognition of plant objects using the methods of graph theory and fractal geometry [12-14].

A plant object can be assessed by its formalized description. Graph theory methods can be used. In this regard, it is necessary to build a graph model that describes the structure of the object. And to carry out the recognition of a plant object not as a whole, but by the characteristic elements of the plant, which are characteristic only of this plant.

Thus, it is possible to recognize an object by the first blocks of an almost quasi-diagonal matrix of edges and a matrix of nodes of the graph-model of a plant object (Fig. 4). In the matrix of edges, blocks 1 and 2 have the same shape, which is characteristic only for grain crops (Fig. 5, a). And in the matrix of nodes, blocks 1 and 2 differ, but in subsequent blocks their shape is similar to the shape of block 2 . It is by this feature that one can distinguish a grain crop from another.

Many leafy plants can be described as a graph with a branched structure. In the matrix of edges, blocks 1 and 2 have the same shape, and in the matrix of nodes, blocks 1 and 2 are different, but in subsequent blocks their shape resembles the shape of block 2 , but on the diagonal the elements resemble the shape of block 2 , and other elements seem to diverge in a similar way from diagonals (Fig. 5, b).

It is necessary to determine the structural patterns of graph models of the object, which will allow solving the problems of finding decision functions and other recognition 
problems. In this case, it is necessary to focus only on the very fact of the presence of structural regularities of a plant object, without describing a specific plant.

This is the most optimal approach. If it is necessary to take into account the geometric parameters of a particular plant, then it is necessary to find out how close the described model of a plant object is to a real object.

The practical application of these models can also be considered in the automation of technological processes for harvesting and processing fruits, where one of the important stages is the recognition of the geometric shape of the object. It is possible to recognize an object by its shadow contour, taking into account its movement [12]. A technique is proposed for determining the shadow contour as an envelope of a family of plane curves obtained when the surface of a recognized object intersects with planes perpendicular to the scanning beam of a system with optoelectronic sensors [13].

The equation of the model surface in a moving coordinate system is determined by the function $f(x, y, z)=0$ and in a fixed coordinate system - $f(X, Y, Z)=0$ and the coordinates of the system OXYZ depend on the coordinates of the system Oxyz, taking into account the direction cosines. In solving this problem, we obtain a family of plane curves. The equation of the shadow contour envelope is obtained by excluding the parameter from the following system: $f(X, Y, H)=0$ and $f_{H}(X, Y, H)=0$. Note that for all plant volumetric objects, the shadow contour is a closed curve. It is possible to consider that the object is moving in space and it distorts the shadow loop. For leafy materials, it is better to use and other recognition methods.

\section{Conclusion}

Research in the field of modeling plant objects of seeds and fruits have shown that it is necessary to construct mathematical models of these objects involved in the technological processes of harvesting and processing agricultural products.

These models should take into account the morphological characteristics of fruit seeds, it is necessary to choose the degree of closeness to the real object and enter the necessary information on the geometric parameters into the surface equations.

The use of mathematical models of plant objects in the automation of technological processes reveals the possibilities of creating more advanced and fundamentally new processes in the development of automatic means and technological lines for harvesting and processing fruits, improving the working bodies of agricultural machines, harvested crops, as well as increasing the efficiency of technological processes for crushing leafy materials.

The further development is expedient to conduct in the following areas: development of specific technological processes, creation of software simulation of complex processes, processing of agricultural materials. The choice of mathematical methods depends on the specific technological process, the set research goal and the capabilities of the software. The possibility of fractal geometry and graph theory allow us to construct the plant model with complex geometry $[9,15]$.

\section{References}

1. Y. Guo, T. Fourcaud, M. Jaeger, X.P. Zhang, B.G Li, Annals of Botany, 107, 723-727 (2011)

2. V. Brukkin, N. Morozova, Mathematical Modelling of Natural Phenomena, 2, 1-53 (2011)

3. D. Clements, Cambridge University Press (2012) doi: 10.1017/CBO9780511896989 
4. T. Nakano, A.W. Eckford, Cambridge University Press, 71-96 (2013) doi: 10.1017/CBO97811839149693

5. D. Eamus, A. Huete, Q. Yu, Cambridge University Press (2016) doi: 10.1017/CBO9781107286221

6. P.H. Cournede, Y. Chen, Q.Wu, C. Baey, B.Bayol, Mathematical Modelling of Natura Phenomena, 4, 112-130 (2013) doi: 10.1051/mmnp/20138407

7. N. Bessonov, V. Mironova, V. Volpert, Mathematical Modelling of Natura Phenomena, 4, 62-79 (2013) doi: 10.1051/mmnp/20138405

8. J.M. Fraser, Cambridge University Press (2020) doi: 10.1017/CBO9781108778459

9. Z. Liu, Z. Zhang, Cambridge University Press (2011) doi: 10.1017/CBO9780511977169

10. A. Garg, A. Negi, A. Agrawal, International Journal of Computer Application, 4, (2014) doi:10.5120/14970-3157

11. I.A. Mayatskaya, D. S. Zagutin, Recognition of plant objects with a complex geometric shape, Science. Education. Practice: proceedings of the International University Science Forum ,Canada, Toronto, November 11, 2020, 214-222 (2020) doi: 10.34660/INF.2020.87.52.032

12. I.A. Mayatskaya, I.A. Krasnobaev, B.M. Demchenko, Global Science and Innovation: proceedings of the VII International Scientific Conference, 189-195 (2016)

13. I.A. Mayatskaya, 2nd International Conference on Industrial Engineering, Applications and Manufacturing, ICIEAM 2016 - Proceedings, IEEE, 7911577 (2016)

14. C.J. Bishop, Y. Peres, Cambridge University Press (2017) doi: 10.1017/CBO9781316460238

15. V. Latora, V. Nicosa, G. Russo, Cambridge University Press, 1-30 (2017) doi: $10.1017 /$ CBO9781316216002 